\title{
MedienPädagogik
}

Zeitschrift für Theorie und Praxis der Medienbildung

\section{Editorial: Visuelle Methoden in der Forschung}

\author{
Horst Niesyto und Winfried Marotzki
}

Die Bedeutung der Bilder in der öffentlichen Kommunikation hat in den letzten Jahren stark zugenommen. Nicht umsonst spricht William Mitchell in seinem Buch «Picture Theory» (1994) von einem «pictorial turn», der sich an den «linguistic turn» anschliesse. Er konstatiert programmatisch: «we may find that the problem of the twenty-first century is the problem of the image». Betrachtet man den Bereich der erziehungs- und sozialwissenschaftlichen Forschung unter der Perspektive, welche Bedeutung hier visuellem Material zukommt, dann stellt man nüchtern fest, dass in den Bereichen der Datenerhebung wie auch der Datenauswertung in methodologischer wie auch in methodischer Hinsicht Defizite bestehen. Das gilt für qualitative wie auch für quantitative Forschungsmethoden gleichermassen. Die wesentlichen Fortschritte qualitativer Methoden in den letzten dreissig Jahren sind beispielsweise vor allem mit neuen Entwicklungen im Bereich der Interpretation von Texten (Interviews, Gruppendiskussion, ethnografische Verfahren) verbunden. Sie stehen im Zusammenhang mit dem «linguistic turn» in den Sozialwissenschaften (konversations- und narrationsanalytische Auswertungsverfahren) und gehen einher mit einer Marginalisierung der Interpretation visueller Dokumente. Bilder wurden wesentlich auch als Texte gesehen («Die Welt als Text»).

Artikulation und kommunikative Verständigung vollziehen sich aber nicht nur im Medium der Sprache und des Textes, sondern auch in demjenigen des Bildes bzw. bewegter Bilder (Film). Die Methoden zum Sprach- und Textverstehen sind relativ gut ausgearbeitet, die Methoden zur Film- und Bildinterpretation sind es im Kontext sozialwissenschaftlicher Forschung nicht. Natürlich gibt es ausgearbeitete Traditionen für die Bildinterpretation im Bereich der Kunstwissenschaft und für Filminterpretation im Bereich der Filmwissenschaft, aber hier liegen andere Fragestellungen zugrunde. Seit einigen Jahren hat eine stärkere Hinwendung zu visuellen Materialien stattgefunden. Inspiriert durch Modelle der Kunst- und Filmwissenschaft, der Medienwissenschaft und der Cultural Studies gibt es jetzt auch im deutschsprachigen Raum erste sozial-, erziehungs- und medientheoretische Versuche, visuelles Material in Forschungskontexten methodisch ernster zu nehmen. Ausdruck davon sind Publikationen wie das Handbuch «Foto- und Filmanalyse in der Erziehungswissenschaft» (Ehrenspeck/Schäffer 2003), die Tagungsdokumentation «Selbstausdruck mit Medien: Eigenproduktionen mit Medien als Gegenstand der Kindheits- und Jugendforschung» (Niesyto 2001) oder verschiedene Beiträge im Online-Magazin «MedienPädagogik» über «Methodologische Forschungsansätze» (Ausgabe 1/2001).

Niesyto, Horst, und Winfried Marotzki. 2005. «Editorial: Visuelle Methoden in der Forschung». MedienPädagogik 9, (Juni), i-vi. https://doi. org/10.21240/mpaed/09/2005.06.08.X. 
Begonnen hatte dieser Prozess insbesondere in der Jugendforschung. So öffneten sich Teilbereiche der Jugendforschung auch für visuelle Methoden der Erhebung und Dokumentation. Zu erwähnen sind in diesem Zusammenhang u.a. Foto-Portraits im Rahmen der Shell-Jugendstudie von 1992, einzelne Projekte im Rahmen des DFG-Schwerpunktprogramms «Pädagogische Jugendforschung» (1980-1986) sowie Projekte der medienpädagogischen Praxisforschung auf der Basis von Eigenproduktionen mit Video (z.B. Projekt «VideoCulture - Video und interkulturelle Kommunikation»). Diese Eigenproduktionen können als Forschungsdaten genutzt werden; es lassen sich über sie auch weitere verbale Äusserungen anregen. Vor allem dann, wenn die sprachlichen Kompetenzen der Subjekte gering bzw. noch wenig ausgeprägt sind (Kinder, Migranten, Menschen aus benachteiligenden sozialen Milieus), ist es wichtig, non-verbale Äusserungsformen anzubieten (vgl. das aktuelle EU-Projekt "Chicam». In einer Zeit, in der Wahrnehmung und Welterleben von Kindern und Jugendlichen stark von Medienerfahrungen geprägt sind, eröffnet Forschung auf der Grundlage von Eigenproduktionen einen ergänzenden bzw. alternativen Zugang zu deren Lebenswelten.

Die aktuelle Online-Ausgabe «Visuelle Methoden in der Forschung» knüpft an diesen Forschungsarbeiten und Diskussionen an und stellt mehrere Ansätze und Projektbeispiele für methodologische und methodische Reflexionen und Modelle zur Arbeit mit Visuellem und zur Interpretation solcher Materialien vor. Die Beiträge spiegeln unterschiedliche disziplinäre, thematische und methodische Zugänge zu visuellen Materialien wider und bieten zahlreiche Anregungen für Forscher/innen. Unabhängig von spezifischen Fragestellungen und Zugängen lassen sich drei Themenfelder benennen, die in unterschiedlicher Intensität und Breite in den Beiträgen deutlich werden:

\section{Die Relevanz von medialen Dokumentationen und Eigenproduktionen als neuer Zugang zum Welterleben von Kindern und Jugendlichen}

DAVID GAUNTLETT (University of Bournemouth, UK) betont in seinem Beitrag «Using creative visual research methods to understand media audiences» vor allem die heuristische Dimension visueller Materialien und die Bedeutung künstlerisch-kreativer Ausdrucksformen, um einen Zugang zu Lebenswelten von Kindern und Jugendlichen zu erhalten und diese zu verstehen. Er plädiert für ethnografische und explorative Vorgehensweisen, die sich auf diese Ausdrucksformen und ihre Entstehungsprozesse einlassen («avoiding linearity») und nach den «mental pictures» in der Weltaneignung junger Menschen fragen. GAUNTLETT referiert eine breite Palette von künstlerisch-kreativen Ausdrucksformen und intendiert mit seinem Ansatz auch ein besseres Zusammenwirken von Forschung und Praxis. 
HEINZ MOSER (Pädagogische Hochschule Zürich) rekurriert in seinem Artikel «Visuelle Forschung - Plädoyer für das Medium «Fotografie» u.a. auf GAUNTLETTS Argumentation, dass visuelle Erfahrungen heute immer wichtiger für den Alltag der Menschen werden: «Wenn man deshalb auf einer visuellen Ebene operiere, spiegelten diese visuellen beziehungsweise kreativen Methoden letztlich die visuelle Natur der heutigen Medien.» Moser entwickelt in seinem Beitrag systematisch die Möglichkeiten visueller Forschung und konzentriert sich dabei auf das Medium Fotografie in ethnografischen Kontexten. Im Mittelpunkt steht das Konzept der «photo elicitation», das unter verschiedensten Labels seit langem vor allem in der Visuellen Soziologie eingesetzt wird. Der methodische Reiz liegt insbesondere in den Möglichkeiten, «räumliche Elemente einer Bildsprache mit den verzeitlichten und chronologisch aufgebauten narrativen «Geschichten` derjenigen, die fotografiert haben, zu verknüpfen» (MOSER). Diese Möglichkeiten werden am Beispiel von Kinderzimmer-Fotografie und Fotoberichten aus einem Züricher Migrationsprojekt konkretisiert.

\section{Die Reflexion methodischer Möglichkeiten, visuelle Ausdrucksformen und Aneignungsprozesse adäquat zu dokumentieren, zu beschreiben und zu ana- lysieren}

GEORG PEEZ (Universität Duisburg-Essen) rückt im Beitrag «Im Foto ist alles gleichzeitig» die Frage ins Zentrum, wie sich die Simultaneität einer Fotografie und die Sequenzialität eines sprachlichen Textes aufeinander beziehen lassen. Er setzt sich mit verschiedenen Verfahren auseinander, die darauf abzielen, Bilder - insbesondere Fotografien - sequentiell zu erschliessen, um sie einer sprachlichen Interpretation zugänglich zu machen. Unter Bezug auf die Aufzeichnungsmöglichkeit visueller Abtastbewegungen, wie sie seit geraumer Zeit in der Neuropsychologie, der Medienwirkungs- und der Medienmarktforschung praktiziert werden, sieht PEEZ in Anlehnung an den Soziologen THOMAS LOER methodische Anschlussmöglichkeiten für eine objektiv hermeneutische Bildanalyse (sukzessive Erschliessung von Bildräumen, Ermittlung «ikonischer Pfade» im Bild).

Auch ULRIKE STUTZ (Kunstpädagogin, Berlin) geht es in dem Beitrag über «Ästhetische Annäherungen an Bilder in der qualitativen empirischen Forschung» um das Wechselspiel von Wahrnehmung und begrifflicher Verarbeitung, insbesondere unter Einbeziehung von ästhetischem Handeln. Ausgehend von einer rezeptionsästhetischen Bildinterpretationsmethode und einem handlungsorientierten Bildauslegungsverfahren skizziert sie am Beispiel eines kunstpädagogischen Foto- und Videoprojekts ästhetische Analysewege (Perspektbildung, Perspektivenwechsel), die auf die Rekonstruktion von Wahrnehmungsprozessen und das Neuentdecken weiterer Deutungsmöglichkeiten abzielen. 
SIEGLINDE JORNITZ und STEFANIE KOLLMANN (Deutsches Institut für Internationale Pädagogische Forschung, Frankfurt/Main) stellen an Beispielen aus der pädagogischen Bilddatenbank «Pictura Paedagogica Online» (DFG-Projekt) Möglichkeiten der systematischen Erschliessung von Bildern unter thematischen Aspekten vor. «Ins Bild hinein und aus dem Bild heraus» - unter diesem Titel fassen die Autorinnen ihre Erfahrungen mit der begrifflichen Verschlagwortung von Bildern zusammen. Am Beispiel des Themenbereichs «Spiel» wird aufgezeigt, wie eine Bilddatenbank als Quelle für eine historisch-pädagogische Forschung genutzt werden kann. Die gewählten Beispiele verdeutlichen den Eigenwert von Bildern gegenüber dem Text, der über bloss illustrierende Funktionen hinausgeht: Visuelle Aneignungs- und Zugangsprozesse eröffnen - entgegen einer meist textlichen Eindeutigkeit - vielfältigere Deutungsmöglichkeiten von Situationen.

STEFAN ISKE und CHRISTIAN SWERTZ (Universität Bielefeld/Universität Wien) beziehen sich in ihrem Beitrag über «Methodologische Fragen der Verwendung von Bild-, Ton- und Textdaten zur Navigationsanalyse» auf aktuelle Forschungen im Bereich E-Learning. Ihre Ausgangsthese ist, dass die Untersuchung der Aneignungsprozesse von Lernenden während des E-Learning in der Forschung bislang unberücksichtigt bleibt. In methodologischer Perspektive entwickeln die Autoren eine Datentriangulation, die auf der audiovisuellen und digitalen Dokumentation von Aneignungsprozessen in hypermedialen Lernumgebungen beruht und eine weitergehende Rekonstruktion der Aneignungsprozesse möglich macht, als dies durch die Beschränkung auf Text- oder Tonaufzeichnungen möglich ist.

\section{Die konzeptionelle Entwicklung und forschungspraktische Erprobung von Video-Dokumentationen und computergestützten Video-Analysen}

SVEN KOMMER und RALF BIERMANN (Pädagogische Hochschule Freiburg) bieten in ihrem Beitrag «Video(technik) in der erziehungswissenschaftlichen Forschung» einen Überblick über den methodologisch-methodischen Entwicklungsstand und geben konkrete Einblicke in das Projekt «Medienbiografien mit Kompetenzgewinn», das aktuell im Rahmen des Kompetenzzentrums für Genderforschung und Bildungsfragen (PH Freiburg) durchgeführt wird. Ausgehend von der Annahme, dass die ausschliessliche Nutzung von verbalen Selbstexplikationen in qualitativen Interviews nicht ausreicht, um die medialen Handlungspraxen der Befragten adäquat zu erfassen, stellen die Autoren ein Triangulations-Konzept vor, das wesentlich auf der videogestützten Beobachtung von Computerkursen beruht. Über rein methodologische Erörterungen hinausgehend werden die einzelnen Schritte der Datenerhebung sowie die Aufgaben bei der quantitativen Kodierung des aufgenommenen Videomaterials sehr konkret auf dem Hintergrund der Projekterfahrungen beschrieben. Die detaillierte Darstellung der Möglichkeiten der computergestützten Filmanalyse-Programme «Catmovie 
und Videograph», die für die Auswertung umfangreichen Materials geeignet sind (u.a. SPSS-Files), vermittelt eine Reihe forschungspraktischer Erfahrungswerte, die für die Planung eigener Vorhaben dienlich sein können.

In einem Fazit-Kapitel halten KOMMER und BIERMANN im Hinblick auf die analysierten Befunde fest, dass sich erst über die Integration der Videodaten Erkenntnisse ergaben, die bei den Interviews von den Befragten nicht expliziert wurden und auch in einer vertiefenden Interpretation dieser Interviews nur bedingt herausgearbeitet werden konnten.

PHILIPP MAYRING (Universität Klagenfurt), MICHAELA GLÄSER-ZIKUDA und SASCHA ZIEGELBAUER (Pädagogische Hochschule Ludwigsburg) stellen in ihrem Beitrag «Auswertung von Videoaufnahmen mit Hilfe der Qualitativen Inhaltsanalyse» ein Projektbeispiel aus der Unterrichtsforschung vor, das den Einfluss emotionaler Faktoren auf schulische Lernprozesse untersucht. Um die emotionale Befindlichkeit analysieren zu können, wurden Emotionen wie Langeweile, Freude oder Angst von Schülerlnnen nicht nur nicht nur mit Hilfe von Befragungen erfasst, sondern darüber hinaus Videomitschnitte von Unterrichtsstunden angefertigt. Der Beitrag skizziert zunächst verschiedene Methoden und Instrumente zur videobasierten Unterrichtsbeobachtung, um dann am Beispiel der qualitativen Video-Inhaltsanalyse von Schüleremotionen detailliert das methodische Vorgehen unter Einbeziehen des Programms Videograph darzustellen.

In der Diskussion ihrer methodischen Erfahrungen heben die AutorInnen hervor, dass die Logik der "Qualitativen Inhaltsanalyse» (MAYRING) auch für Beobachtungsstudien gut anwendbar sei: Qualitative Video-Inhaltsanalyse versuche theoriegeleitet Kategorien zu definieren, genaue Zuordnungsregeln und Analyseablaufmodelle festzulegen und diese mit der Beobachterübereinstimmung zu überprüfen. Erst in der Zusammenschau von verbal und audio-visuell erhobenen Daten ergebe sich ein schlüssiges Bild der emotionalen Befindlichkeit der untersuchten Person.

LARS GERHOLD und STEFAN BORNEMANN (Universität Kassel) stellen in ihrem Beitrag über "Qualitative Analyse audiovisueller Informationen mit ATLAS.ti» dar, weshalb das Videoanalyse-Programm ATLAS.ti aus ihrer Sicht besonders geeignet ist, um filmisches Material interpretativ zu erschliessen. Hervorgehoben werden unter Verweis auf die konzeptionelle Anbindungsmöglichkeit an den Analyseansatz der Grounded Theory nach GLASER/STRAUSS - vor allem die einzelnen Komponenten der hermeneutischen Einheit (Primärdokumente, Kodes, Zitate und Memos). Die enge Verbundenheit von Analyse, Thesen- und Theorieentwicklung sei inhärenter Bestandteil sowohl der Grounded Theory als auch im strukturgebenden Umgang mit der Software ATLAS.ti. An einem Fallbeispiel aus der Nachrichtensendung «Wochenspiegel» stellen die Autoren die einzelnen Schritte des Kodierprozesses sowie die Entwicklung von Netzwerken vor. Abschliessend werden Vor- und Nachteile diskutiert und ein Ausblick auf mögliche Einsatzfelder der Filmanalyse mit ATLAS.ti gegeben. 
Neben den genannten Beiträgen erreichten uns weitere Artikel, die zwar auf Visualität eingingen, jedoch aus unterschiedlichen Gründen nicht zu den Intentionen des «Call for Papers» passten. Insgesamt zeigte sich ein starkes Interesse am Thema «Visuelle Methoden in der Forschung». Erfreulich ist die zunehmende Öffnung für interdisziplinär angelegte Diskurse und Projekte. Visuelle Methoden werden in den letzten Jahren in wachsendem Umfang in unterschiedlichen Forschungskontexten angewendet. Auch wenn die methodologische Diskussion und der Austausch über forschungspraktische Erfahrungen beim Einsatz visueller Methoden - gerade im Bereich computergestützter Dokumentations- und Analyseverfahren - erst begonnen haben, so zeigen doch verschiedene Beiträge in dieser Ausgabe des Online-Magazins: die Erhebung und die Zusammenschau von verbalen und nicht-verbalen Daten ist notwendig, um umfassendere Zugänge zum Welterleben von Kindern und Jugendlichen zu bekommen.

\section{Literatur}

Ehrenspeck, Yvonne / Schäffer, Burkhard (Hrsg.): Foto- und Filmanalyse in der Erziehungswissenschaft. Opladen 2003.

Mitchell, William: Picture Theory: Essays on Verbal and Visual Representation. University of Chicago Press 1994.

Niesyto, Horst (Hrsg.): Selbstausdruck mit Medien: Eigenproduktionen mit Medien als Gegenstand der Kindheits- und Jugendforschung. München 2001. 